\title{
In vivo and in vitro propagation of intraductal papillary mucinous neoplasms
}

\author{
Hirohiko Kamiyama ${ }^{1,4}$, Mihoko Kamiyama ${ }^{1,4}$, Seung-Mo Hong ${ }^{1}$, Collins A Karikari ${ }^{1}$, Ming-Tseh Lin ${ }^{1}$, Michael W Borges ${ }^{1}$, \\ Margaret Griffith ${ }^{1}$, Angela Young ${ }^{1}$, Alexis Norris-Kirby ${ }^{1}$, Conrad Lubek', Masamichi Mizuma ${ }^{1}$, Georg Feldmann ${ }^{1}$, \\ Chanjuan Shi ${ }^{1}$, Hong Liang ${ }^{1}$, Michael G Goggins ${ }^{1,2}$, Anirban Maitra ${ }^{1,3}$, Ralph H Hruban ${ }^{1,3}$ and James R Eshleman ${ }^{1,3}$
}

Intraductal papillary mucinous neoplasms (IPMNs) are one of the three known curable precursor lesions of invasive pancreatic ductal adenocarcinoma, an almost uniformly fatal disease. Cell lines from IPMNs and their invasive counterparts should be valuable to identify gene mutations critical to IPMN carcinogenesis, and permit high-throughput screening to identify drugs that cause regression of these lesions. To advance the study of the biological features of IPMNs, we attempted in vivo and in vitro growth of selected IPMNs based on the hypothesis that IPMNs could be grown in the most severely immunodeficient mice. We examined 14 cases by implanting them into nude, severe combined immunodeficient (SCID), and NOD/SCID/IL2R $\gamma^{\text {null }}$ (NOG) mice, in addition to direct culture, to generate tumor xenografts and cell lines. One sample was directly cultured only. Thirteen tumors were implanted into the three types of mice, including 10 tumors implanted into the triple immunodeficient NOG mice, in which the majority (8 of 10) grew. This included five IPMNs lacking an invasive component. One of the explanted IPMNs, with an associated invasive carcinoma, was successfully established as a cell line. Tumorigenicity was confirmed by growth in soft agar, growth in immunodeficient mice, and the homozygous deletion of $p 16 / c d k n 2 a$. Epithelial differentiation of the cell line was documented by cytokeratin expression. Patient origin was confirmed using DNA fingerprinting. Most non-invasive IPMNs grow in NOG mice. We successfully established one IPMN cell line, and plan to use it to clarify the molecular pathogenesis of IPMNs.

Laboratory Investigation (2010) 90, 665-673; doi:10.1038/labinvest.2010.51; published online 15 March 2010

KEYWORDS: cell lines; immunodeficient mice; intraductal papillary mucinous neoplasm (IPMN); pancreatic cancer; precursor lesions

Pancreatic ductal adenocarcinoma (PDAC) is a notorious deadly cancer that affected an estimated 37680 Americans in 2008, and resulted in approximately 34290 deaths (case: fatality ratio $=91 \%) .{ }^{1}$ Approximately 230000 patients per year develop PDAC worldwide and the 5-year survival rate for these patients is expected to be only $4 \%{ }^{2,3}$ Effective early detection and treatment can improve these statistics, but require a full understanding of the molecular biology of the precursor lesions that give rise to invasive cancer. There are three documented morphologic precursors to pancreatic cancer: pancreatic intraepithelial neoplasias (PanINs), intraductal papillary mucinous neoplasms (IPMNs), and mucinous cystic neoplasms. ${ }^{4}$
IPMNs are mucin-producing epithelial neoplasms that, by definition, involve the main and/or branch pancreatic ducts and often, although not always, have a papillary architecture. ${ }^{4,5}$ It is clear that some IPMNs progress to invasive adenocarcinoma over time ${ }^{6-9}$ however, several fundamental unanswered questions remain. The complete molecular pathogenesis, of invasive pancreatic cancers arising from IPMNs, is not established, as it is for those arising from PanINs. ${ }^{10,11}$ For example, although the progressive accumulation of mutations in the KRAS2, TP53, p16/cdkn2a, and DPC4 genes has been well established for PanINs, a similar progression is not as well delineated for the various subtypes of IPMNs. ${ }^{12-17}$ In addition, different genes are sometimes

\footnotetext{
'Department of Pathology, The Johns Hopkins University School of Medicine, Baltimore, MD, USA; ${ }^{2}$ Department of Medicine, The Johns Hopkins University School of Medicine, Baltimore, MD, USA and ${ }^{3}$ Department of Oncology, The Sol Goldman Pancreatic Cancer Research Center, The Johns Hopkins University School of Medicine, Baltimore, MD, USA

Correspondence: Dr JR Eshleman, MD, PhD, Department of Pathology, The Johns Hopkins University, Suite 341, David Koch CRB-II, 1550 Orleans Street, Baltimore, MD 21231 USA.

${ }^{4}$ These two authors contributed equally to this work.
}

Received 1 July 2009; revised 22 December 2009; accepted 28 December 2009 
targeted in PanINs and IPMNs. For example, the loss of the STK11 gene is observed in up to one-third of IPMNs, but is rarely found in PanINs and PDACs. ${ }^{18}$ Similarly, activating mutations of the PIK3CA gene have been observed in IPMNs, but not in PanIN lesions. ${ }^{19}$ The natural history of IPMNs, such as the time and frequency of progression to PDAC, is also not well defined. ${ }^{9,20,21}$ Although the size of the lesion is associated with progression, it is unclear whether a specific size can be used as a clinical cutoff for surgical resection, although one has been proposed. ${ }^{22}$ In addition, no model exists in which to evaluate potential chemopreventative agents. IPMN cell lines would be valuable to clarify these issues. Thus, we used the techniques that have been used to establish cell lines from invasive pancreatic cancers to attempt to make IPMN cell lines. ${ }^{23}$

In this report, we used triple immunodeficient NOG mice to propagate IPMNs, the majority of which grew. From one of these xenografted tumors, we aspirated the fluid from the cystic component, harvested the solid component separately, and generated cell lines from both of them. We conclude that IPMNs can be grown both in vivo and in vitro provided that the mice are sufficiently immunodeficient.

\section{MATERIALS AND METHODS}

\section{Patients, Histopathology, and Tissue Harvest}

On the basis of frozen section diagnoses, surgically resected samples were classified as IPMNs with or without an associated invasive carcinoma. Frozen section diagnoses were also confirmed with permanent sections. This work was performed with human subjects and animal committee approval. Freshly harvested IPMNs were implanted into mice and/or directly cultured within $2 \mathrm{~h}$ of resection.

\section{In Vivo Growth as Mouse Xenografts}

IPMNs were subcutaneously implanted in nude, severe combined immunodeficient (SCID) $\left(p r k d c^{\text {null }}\right)$, or NOG $\left(\mathrm{NOD} / P r k d c^{\text {null }} / \mathrm{IL}-2 \mathrm{R} \gamma^{\text {null }}\right)$ mice. Mice were monitored at regular intervals and killed when tumors reached about $1 \mathrm{~cm}^{3}$. Tumors were removed under sterile conditions and used for reimplantation, cryopreserved in DMSO, fixed in formaldehyde, and plated for tissue culture growth.

\section{In Vitro Cell Culture}

In a laminar flow biosafety cabinet, tumors explanted from mice or harvested directly in the surgical pathology suite were finely minced $(<2 \mathrm{~mm})$ and digested using minimum essential medium (MEM, GIBCO, Carlsbad, CA, USA) containing collagenase type $1(1 \mathrm{mg} / \mathrm{ml}$, GIBCO) and hyaluronidase $(0.7 \mathrm{mg} / \mathrm{ml}$, Sigma-Aldrich, St Louis, MO, USA). The single cells were cultured as described earlier, ${ }^{23}$ with the following modifications. Cells were plated on $25 \mathrm{~cm}^{2}$ flasks coated with rat-tail type 1 collagen (BD Biosciences, San Jose, CA, USA) and maintained in MEM containing $20 \%$ fetal bovine serum (FBS, GIBCO), 1\% penicillin-streptomycin (GIBCO), $5 \mathrm{ng} / \mathrm{ml}$ EGF (GIBCO), and $0.2 \mathrm{U} / \mathrm{ml}$ human recombinant insulin (GIBCO). In addition, to screen the optimal growth condition for each tumor, we varied several combinations for both the medium and substrates on 24-well plates for five cases.

To overcome fibroblast overgrowth, some cultures were treated periodically with selective trypsinization to remove the fibroblasts. Using a phase microscope, fibroblast-rich and tumor-rich regions were identified and marked on the bottom of the flask. Trypsin was added at room temperature and the culture was monitored. The reaction was stopped when the unwanted cells had detached, whereas the tumor cells were still attached, by aspirating the supernatant and quenching the trypsin by the addition of complete media.

\section{Characterization of Cell Lines \\ Immunohistochemistry}

Immunohistochemistry was used to detect the expression of cytokeratin (AE1 + AE3) (Ventana, Tucson, AZ, USA, PCK26 mouse monoclonal, predilute), vimentin (Ventana, V9 mouse monoclonal, predilute), MUC1 (Novocastra, New Castle on Tyne, UK, Ma552 mouse monoclonal, 1:200), MUC2 (Santa Cruz Biotechnology, Santa Cruz, CA, USA, H-300 rabbit polyclonal, 1:200), p16/CDKN2A (CINtec. Inc, Westborough, MA, USA, E6H4, mouse monoclonal, predilute), TP53 (Dako, Carpinteria, CA, USA, DO-7 mouse monoclonal, predilute), and DPC4/SMAD4 (Santa Cruz Biotechnology, B-8 mouse monoclonal, 1:200). The expression patterns in cell lines were compared with those observed in the primary IPMN and its xenograft. All the immunohistochemical reactions, except DPC4/SMAD4, were performed on a Ventana Benchmark XT, and all detection kits were from Ventana. Labeling for DPC4/SMAD4 protein was performed on a Dako autostainer using the DAKO Envision Plus detection kit.

\section{Sequencing and multiple ligation-dependent probe amplification}

The protein coding exons of Kras, TP53, p16/CDKN2A, and DPC4/SMAD4, in addition to exons 9 and 20 of the PIK3CA gene, were sequenced in the IPMN-1T and IPMN-1Asp cell lines, as described earlier. ${ }^{11}$ Multiple ligation-dependent probe amplification (MLPA) was performed for p16/ CDKN2A and DPC4/SMAD4 using the SALSA MLPA kit (MRC-Holland, Amsterdam, Netherlands) as per manufacturer's instructions, and products were resolved on a Beckman-Coulter CEQ 8000 capillary electrophoresis instrument.

\section{Fingerprinting}

Microsatellite DNA fingerprinting was performed using the PowerPlex 1.2 system (Promega Corporation, Madison, WI, USA) at the Johns Hopkins University DNA core facility. The germline pattern was established using normal duodenum from the patient. 


\section{Soft agar assay}

Anchorage independence was tested using an agar cloning assay with $0.5 \%$ agar with 100000 suspended cells on $1 \%$ hard agar in six-well plates using six replicates for each sample. Spherical colonies, whose size was $>50 \mu \mathrm{m}$, were counted in 10 random fields using phase microscopy weekly. After 4 weeks, colonies were stained with crystal violet. HPDE is an immortalized normal epithelial pancreatic duct cell line and was used as a negative control. ${ }^{24}$ Pancl is a PDAC cell line and was used as the positive control. ${ }^{25}$

\section{Matrigel invasion assay}

Matrigel invasion assays were performed using the BioCoat matrigel invasion chamber (BD Biosciences). Invasive cell derivatives, from largely non-invasive cells, were selected by plating $2.5 \times 10^{5}$ cells in $2 \mathrm{ml}$ of MEM in the top chamber of a six-well plate and selecting cells from the bottom chamber at various times, as described in Results. The process was repeated to obtain second and third passage invasive cells.

Quantitative measurements were performed in 24-well plates to assess invasive capacity of IPMN-1T cells, IPMN-1T cells twice selected for invasion, and Pancl control cells. For these assays, $2.5 \times 10^{4}$ cells, in $0.5 \mathrm{ml}$ of serum-free MEM, were applied to the top chamber. The lower well, coated with collagen, was filled with complete culture medium containing $20 \%$ FBS. After $6-48 \mathrm{~h}$ of incubation, the non-invasive cells on the upper surface of the filter were removed with a cotton swab. The filters were fixed in methanol, stained with hematoxylin, and the cells were counted under a microscope at $\times 60$ magnification. The cells that had invaded through the matrigel were counted in 10 randomly selected fields, and the count numbers were averaged. The percent invasion was calculated as the number of cells that had invaded through the matrigel divided by the number of cells that had migrated through the filter without matrigel.

\section{Tumor xenograft}

Tumorigenicity in mice was confirmed by subcutaneously injecting approximately 20 million cells, from culture, bilaterally into two mice. Tumor volume (TV) was calculated according to the formula: TV $\left(\mathrm{mm}^{3}\right)=$ length $\times$ width ${ }^{2} \times 0.5$. Explanted tumor xenografts were used for reimplantation and fixed in formalin for histology.

\section{RESULTS}

\section{Case Selection and Primary Tumors}

Owing to the need to fully understand the pathogenesis of IPMNs and their derivative invasive cancers, we attempted in vivo and in vitro propagation of these lesions. We initially attempted to grow IPMNs with and without an invasive component, but after initial success with in vivo propagation, we focused on those IPMNs without an invasive component (Table 1). Nine of 14 patients were male $(64.2 \%)$, and the mean age was 66 years old. Five of the IPMNs that we xenografted included an associated invasive cancer elsewhere in the lesion, and nine of the IPMNs were exclusively noninvasive. The non-invasive IPMN components were classified as high-grade dysplasia in seven cases $(50 \%)$, moderate dysplasia in five $(36 \%)$, and low-grade dysplasia in two (14\%). The associated invasive component was a poorly differentiated adenocarcinoma in one case $(20 \%)$, a moderately differentiated adenocarcinoma in three $(60 \%)$, and a well-differentiated adenocarcinoma in one case $(20 \%)$. The invasive adenocarcinomas were categorized as colloid type in one case and tubular type in four cases.

The results of the IHC labeling for cytokeratin, MUC1, MUC2, p16/CDKN2A, TP53, and DPC4/SMAD4, on the primary neoplasms, are shown in Table 2. Most of the invasive IPMNs were the pancreatobiliary subtype and produced associated tubular carcinomas (cases 1-4). Case 5 was the only intestinal subtype and formed a colloid carcinoma. In contrast, non-invasive IPMNs were either gastric or intestinal subtypes, except for case 8 . Cytokeratin was positive and vimentin was negative for all cases. MUC expression patterns matched with the subtype, in which MUC1 was positive for the pancreatobiliary type, and MUC2 was positive for the intestinal type. ${ }^{26}$ All non-invasive IPMNs expressed p16/CDKN2A and DPC4/SMAD4, but were negative for TP53. The invasive IPMNs showed a loss of p16/ CDKN2A expression in three out of five cases, while TP53 was positive in one out of five cases. All five cases had intact DPC4/SMAD4.

\section{In Vivo Growth of Human IPMNs in Immunodeficient Mice}

We studied 14 total cases, of which only one was directly cultured (Table 3, case 11). Of the 13 IPMNs implanted in mice, one was implanted into a nude mouse, two were implanted into SCID mice, eight were implanted into triple immunodeficient NOG mice (Table 3, cases 2, 3, 4, 5, 6, 7, 9, and 14), and two were implanted into both SCID and NOG mice (Table 3, cases 8 and 12). Of the 13 implanted IPMNs, 11 grew as tumors in mice, whereas 2 did not grow. We attempted to culture, after explanting, 4 of the 11 tumors that grew as xenografts (Table 3, cases $1,5,8$, and 9). The other seven mice died of infection or other causes before their tumors could be harvested.

\section{In Vitro Growth of IPMNs After Explanting from Mice}

After expansion in the immunodeficient mice, we explanted four cases for in vitro growth (Table 3). Another three cases were directly cultured from the surgical pathology suite (two of which were also grown in mice).

For case 1, the xenografted neoplasm formed a cyst. The fluid in the cyst was aspirated and cultured, and the resultant cell line was designated IPMN-1Asp, whereas the solid component of the same neoplasm was cultured and this cell line was designated IPMN-1T. Fibroblasts, which normally 
Table 1 Clinicopathological findings of patients with IPMNs

\begin{tabular}{|c|c|c|c|c|c|c|c|}
\hline Case & Sex & $\mathrm{Age}^{\mathrm{a}}$ & Diag $^{b}$ & Location/size $(\mathrm{cm}) /$ type $^{c}$ & Dysplasia & Invasion $^{d}$ & Pancreatectomy ${ }^{\mathrm{e}}$ \\
\hline 1 & M & $40 \mathrm{~s}$ & IPMN/ml & $\mathrm{Ph} / 2.5 /$ Main & High & Mod/Tub & W \\
\hline 3 & M & $50 \mathrm{~s}$ & IPMN/I & $\mathrm{Ph} / 2.0 /$ Main & High & Mod/Tub & W \\
\hline 4 & M & $80 \mathrm{~s}$ & IPMN/I & $\mathrm{Ph} / 6.0 /$ Main & High & Mod/Tub & W \\
\hline 6 & $\mathrm{~F}$ & $80 \mathrm{~s}$ & IPMN & $\mathrm{Ph} / 3.0 /$ Main & High & - & W \\
\hline 7 & M & $60 \mathrm{~s}$ & IPMN & $\mathrm{Ph} / 3.0 /$ Main & Moderate & - & W \\
\hline 8 & $\mathrm{~F}$ & $80 \mathrm{~s}$ & IPMN & $\mathrm{Ph} / 3.0 /$ Main & Moderate & - & W \\
\hline 9 & $\mathrm{~F}$ & $70 \mathrm{~s}$ & IPMN & $\mathrm{Ph} / 2.5 /$ Main & High & - & W \\
\hline 10 & $\mathrm{~F}$ & $40 \mathrm{~s}$ & IPMN & $\mathrm{Ph} / 1.5 /$ Main & Moderate & - & W \\
\hline 14 & M & $70 \mathrm{~s}$ & IPMN & $\mathrm{Pb} / 6.0 / \mathrm{Branch}$ & Low & - & MP \\
\hline
\end{tabular}

${ }^{\mathrm{a}}$ Age in decade of life.

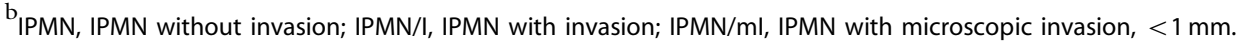

${ }^{\mathrm{C}} \mathrm{Ph}$, pancreas head; $\mathrm{Pb}$, pancreas body; Pt, pancreas tail; Main, main duct type; Branch, Branch duct type.

${ }^{\mathrm{d}}$ Differentiation and type of invasive carcinoma. Mod, moderately differentiated; Poor, poorly differentiated; Well, well differentiated; Tub, tubular type; Coll, colloid type; -, not applicable.

eW, whipple; TP, total; DP, distal; MP, middle.

Table 2 Immunohistochemical profile of primary IPMNs

\begin{tabular}{|c|c|c|c|c|c|c|c|c|}
\hline Case & Invasion & Subtype ${ }^{a}$ & Cytokeratin ${ }^{\mathrm{b}}$ & $M U C 1^{b}$ & $\mathrm{MUC2}^{\mathrm{b}}$ & p16 & $T P 53^{\mathrm{b}}$ & $D P C 4 / S M A D 4$ \\
\hline 1 & Yes/Ml ${ }^{c}$ & PB/ONC & Exp & Exp & No exp & Lost $^{d}$ & No exp & Intact \\
\hline 3 & Yes & PB & Exp & $\operatorname{Exp}^{e}$ & No exp & Lost & No exp & $\operatorname{lntact}^{\mathrm{f}}$ \\
\hline 4 & Yes & GAST/PB & Exp & $\operatorname{Exp}^{9}$ & No exp & Intact & No exp & Intact \\
\hline 7 & No & GAST/INT & Exp & No exp & $\operatorname{Exp}^{9}$ & Intact & No exp & Intact \\
\hline 8 & No & GAST/PB & Exp & $\operatorname{Exp}^{9}$ & No exp & Intact & No exp & Intact \\
\hline 9 & No & INT & Exp & No exp & Exp & Intact & No exp & Intact \\
\hline 10 & No & INT & Exp & No exp & Exp & Intact & No exp & Intact \\
\hline 14 & No & GAST & Exp & No exp & No exp & Intact & No exp & Intact \\
\hline
\end{tabular}

${ }^{\mathrm{a} O N C}$, oncocytic; PB, pancreatobiliary; GAST, gastric; INT, intestinal.

${ }^{b}$ No exp, no expression; exp, expression.

${ }^{\mathrm{c}} \mathrm{Ml}$, microscopic $<1 \mathrm{~mm}$ invasion.

$\mathrm{d}_{\text {Focally lost. }}$

${ }^{\mathrm{e}}$ Non-invasive IPMN was negative, whereas invasive adenocarcinoma was positive.

f Non-invasive IPMN was intact, whereas lost in invasive adenocarcinoma.

${ }^{g}$ Focally positive expression. 
Table 3 Strategy and results of in vivo and in vitro propagation

\begin{tabular}{llll}
\hline Case & Strategy (DC/mouse) & In vivo growth & In vitro growth \\
\hline 1 & Nude & Growth & IPMN-1T, IPMN-1Asp \\
2 & NOG & No growth & NC \\
3 & NOG & Growth & NC \\
4 & NOG & Growth & NC \\
5 & NOG & Growth & IG \\
6 & NOG & Growth & NC \\
7 & NOG & Growth & NC \\
8 & NOG, SCID & Growth & IG \\
9 & NOG & Growth & IG \\
10 & SCID & Growth & NC \\
11 & DC & NI & IG \\
12 & NOG, SCID & No growth & NC \\
13 & DC, SCID & Growth & IG \\
14 & DC, NOG & Growth & IG
\end{tabular}

DC, direct culture; NC, not cultured; IG, initial growth but failed to establish as a cell line; NI, not implanted. overgrow in such cultures, were removed using selective trypsinization. ${ }^{23}$ Neoplastic cells were successfully purified to homogeneity in the first passage for both IPMN-1T and IPMN-1Asp.

We had several problems producing cell lines from the other six cases. In case 5, the neoplastic cells did not attach, although fibroblasts attached and grew. In cases 9 and 11, some neoplastic cells attached and grew initially, but the growth rate was too slow and fibroblasts overgrew the culture in 2 weeks. In cases 13 and 14, neoplastic cells grew poorly and gradually died in primary cultures.

In five cases, we varied the basal media and substrate. Four types of basal medium were used, including MEM, DMEM, RPMI, and a 1:1 (volume:volume) mixture of MEM and RPMI supplemented as described in Materials and methods. For basal medium, the results using MEM and DMEM were equivalent and consistently superior to RPMI. Five different substrates were used: uncoated tissue culture plastic, glass cover slips, tissue culture plastic coated with rat-tail collagen, matrigel, or polylysine. In all cases, rat-tail collagen-coated flasks were much better than matrigel, polylysine, or uncoated flasks. Glass cover slips were the worst substrate. Fibroblast growth seemed to be stimulated on both matrigel
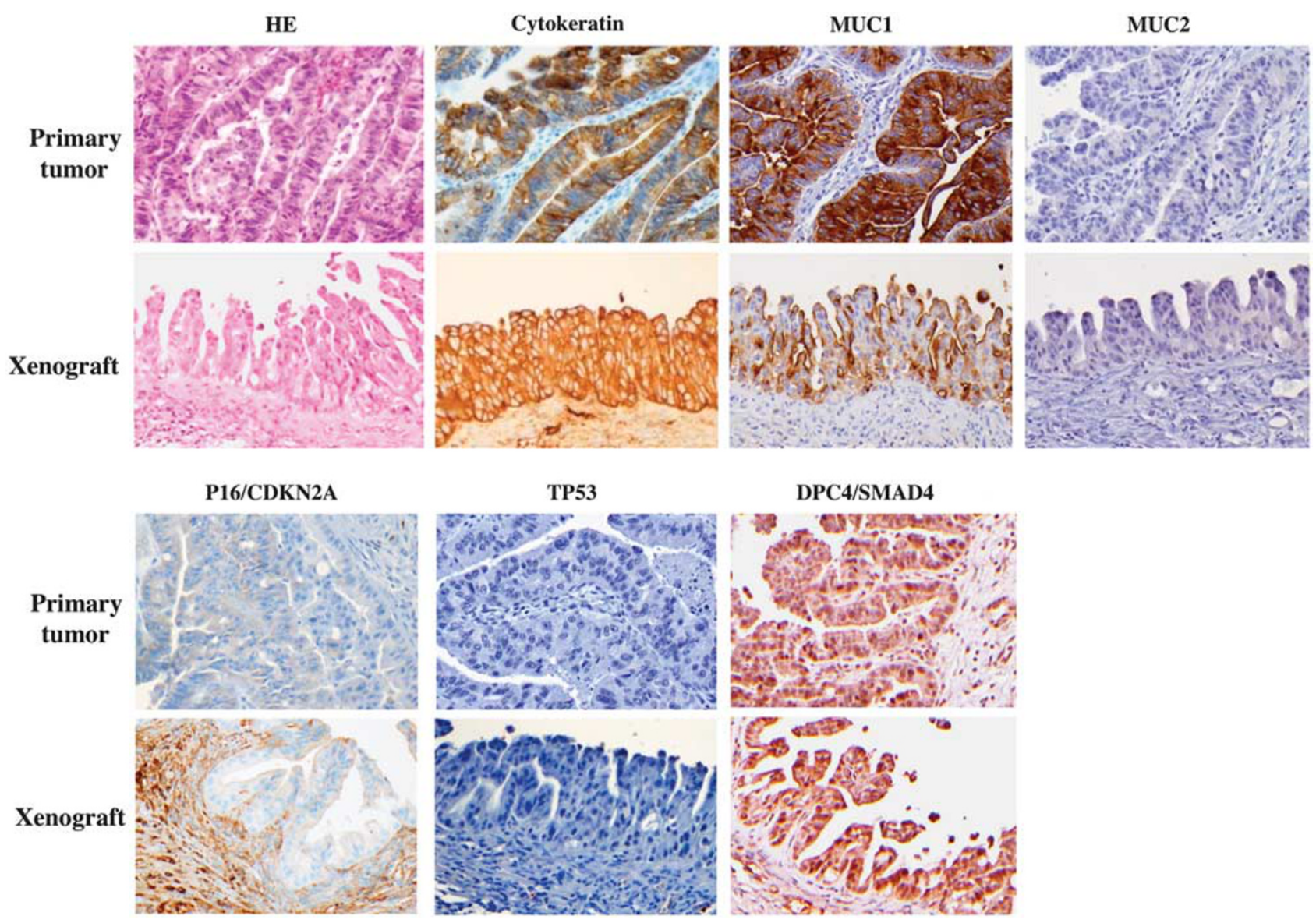

Figure 1 Histology and immunohistochemistry of matched primary tumor and corresponding xenograft for case 1. Hematoxylin and eosin (HE) and immunohistochemical labeling for cytokeratin, MUC1, MUC2, P16/cdkn2a, TP53, and DPC4/SMAD4. Negative region of P16/cdkn2a is shown for the primary tumor; however, the staining is heterogeneous as shown in Supplementary Figure 2. Magnification as indicated. 
and polylysine. Selective trypsinization (see methods) helped to prevent fibroblast overgrowth.

\section{Histological Comparison of Primary Tumors, Xenografts, and IPMN-1 Cell Lines}

For case 1, we compared the histological and immunohistochemical findings in the matched primary tumor, the first passage xenograft, and the cell line (Figures 1 and 2). The primary tumor was a main duct-type IPMN with highgrade dysplasia. The subtype was focal oncocytic mixed with pancreatobiliary. Focally, the neoplastic cells formed small irregular nests in the extensive inflammatory stroma associated with the neoplasm, representing $<1 \mathrm{~mm}$ microscopic invasion. The pathologic stage was T1NOMX. The first passage xenograft grew as an IPMN without invasion. Immunolabeling for cytokeratin was diffusely positive in all three samples (the primary tumor, the xenograft, and the cell line), whereas labeling for vimentin was consistently negative. The primary IPMN expressed MUC1, but did not express MUC2 (Figure 1), and this pattern was maintained in the xenograft and the cell lines (Figure 2). Immunolabeling for the p16/CDKN2A protein was generally lost in the primary tumor, in the xenograft, and in the cell lines, although there was some heterogeneous expression in the primary tumor (see Supplementary Figure S1). The primary IPMN, the xenograft, and the cell lines did not stain with antibodies to the TP53 protein. Expression of the DPC4/SMAD4 protein was positive in the primary tumor, xenograft, and cell lines.

Case 8 was an IPMN with moderate dysplasia and was composed of pancreatobiliary and gastric subtypes. It did not have an associated invasive component. For MUC1, the primary IPMN was focally positive, and the xenograft was consistently positive. For MUC2, both the primary tumor and the xenograft were negative. Immunolabeling for the TP53 protein was negative in the primary tumor and the xenograft. In both the primary tumor and xenograft, P16 and DPC4/SMAD4 were intact (Supplementary Figure S2). We were unsuccessful at establishing a cell line for the explanted cells from case 8 .

\section{Genetic Characterization of IPMN-1 Cell Lines}

Both IPMN-1T and IPMN-1Asp were wild type for Kras and PIK3CA. For TP53, there was only a germline SNP (P72R) and no somatic mutations were detected. For $p 16 / C D K N 2 A$, there was a homozygous deletion as shown by MLPA (Figure 3). This homozygous deletion is consistent with our
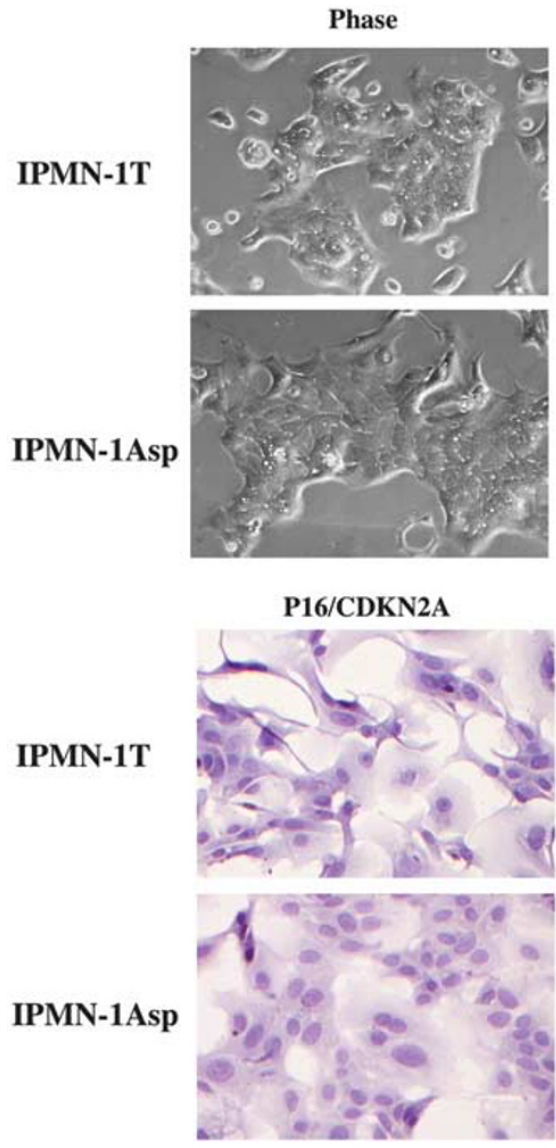

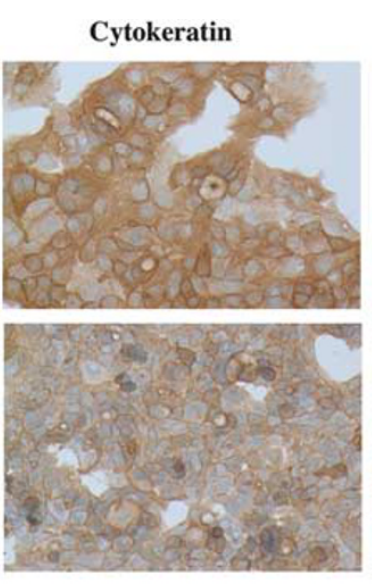

TP53
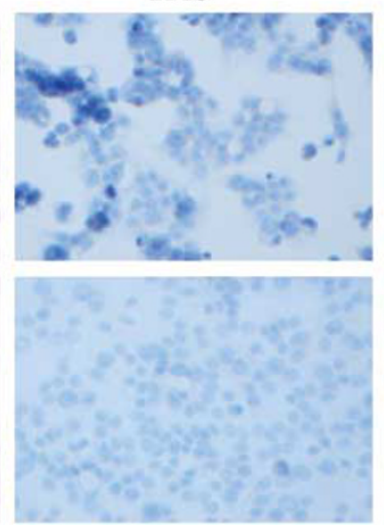
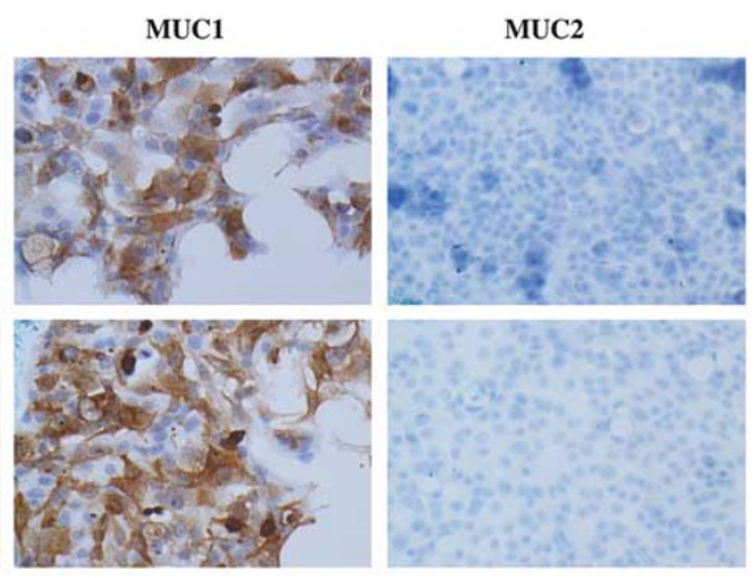

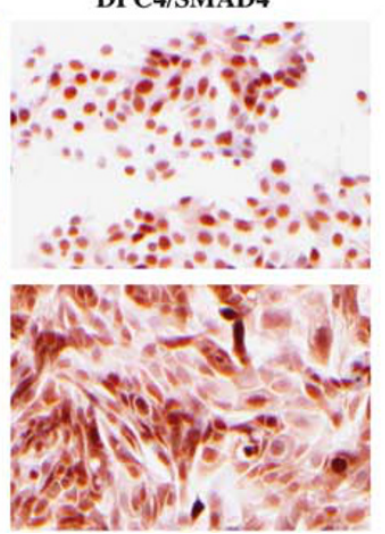

Figure 2 Phase microscopy and immunohistochemistry for the cell lines, IPMN-1T and IPMN-1Asp, stained for cytokeratin, MUC1, MUC2, p16/cdkn2a, TP53, and DPC4/SMAD4 $(\times 20)$. 

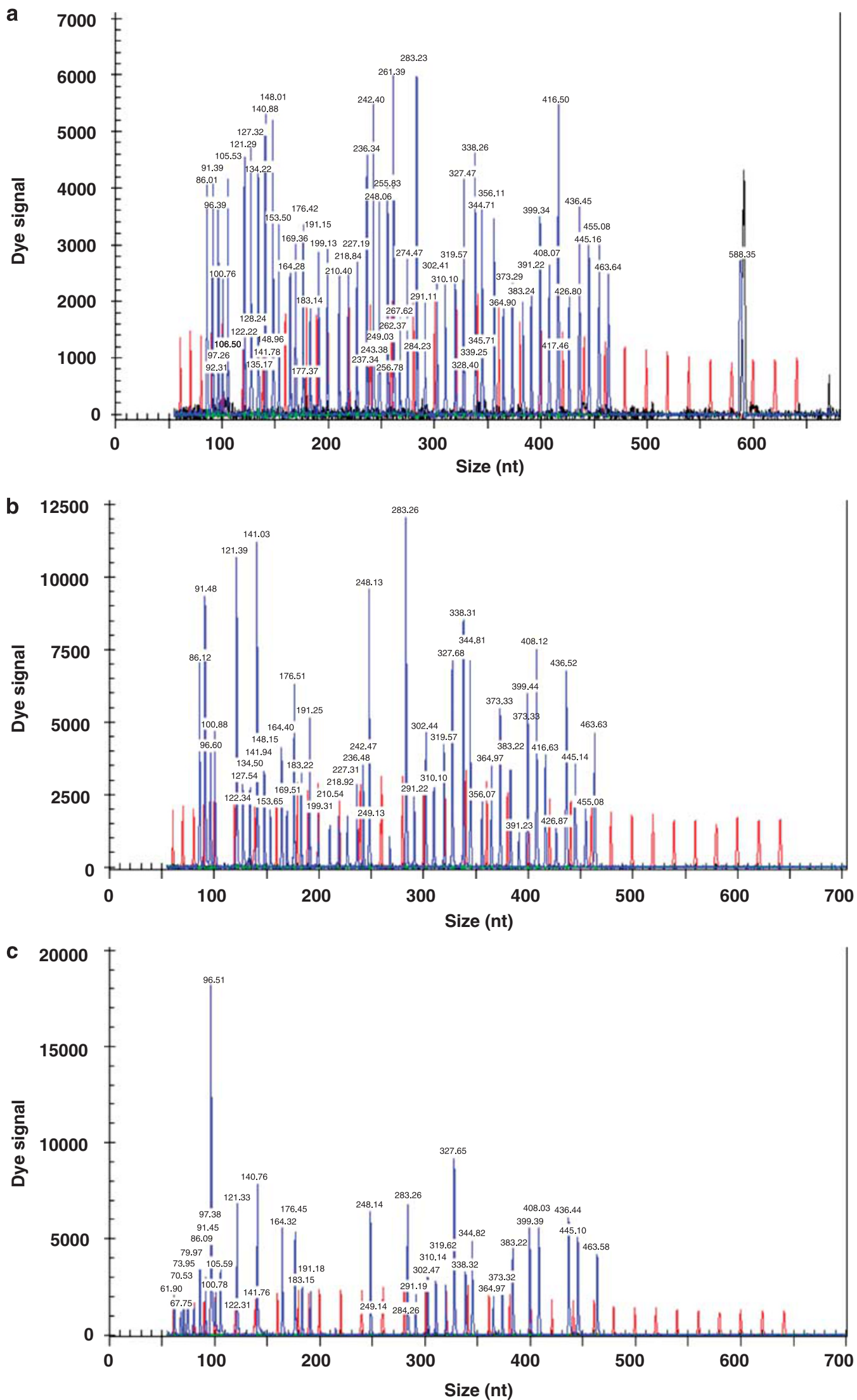

Figure 3 MLPA electropherograms of p16/CDKN2A. Negative wild-type control (a), positive control cell line with a homozygous deletion (b), and IPMN-1T (c). 

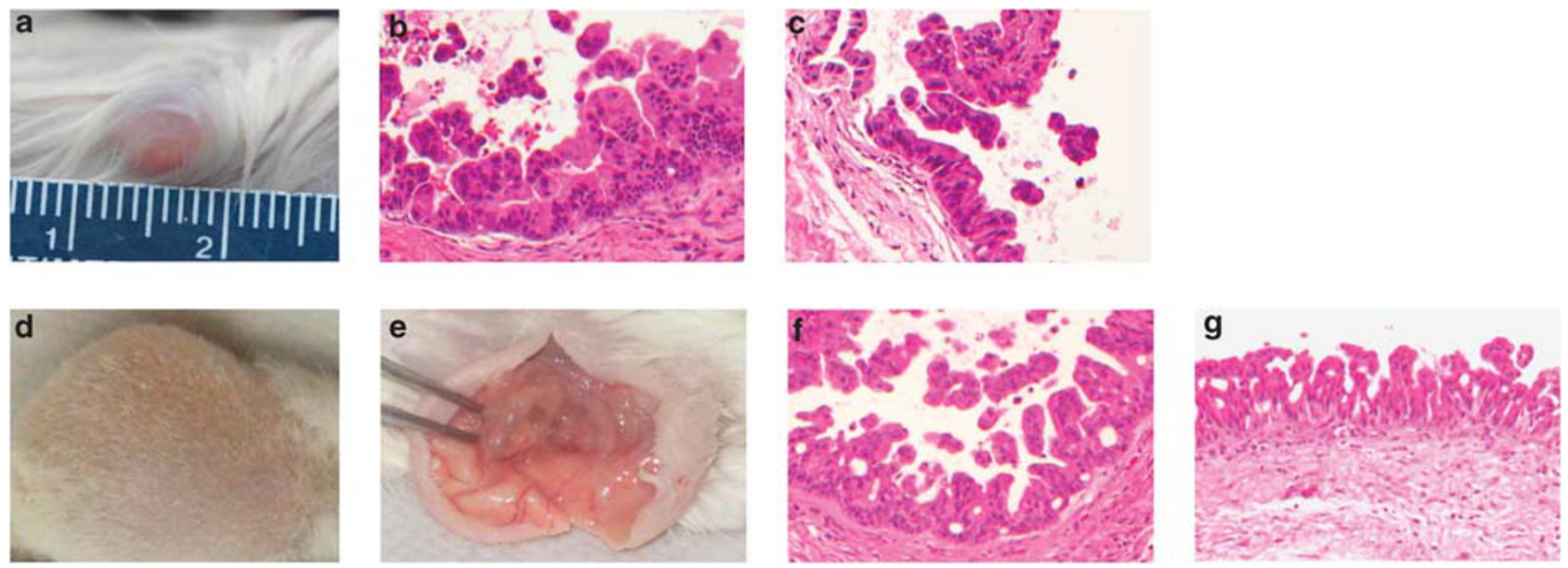

Figure 4 IPMN-1 reimplantation. The tumor in NOG mouse approximately 12 weeks after injection with 20 million IPMN-1T cells (a). Histology of the reimplanted tumor $(\times 20)$ showing a region without invasion $(\mathbf{b}, \mathbf{c})$. The third passage tumor in NOG mouse in 12 weeks after injection $(\mathbf{d})$. Tumor shown in (d) after opening the skin and bisecting the tumor, revealing the nodular features of the tumor (e). Histology of the third passage tumor ( $\times 20)$ without evidence of invasion (f, $\mathbf{g})$.

inability to amplify by PCR any of the exons, despite multiple attempts, and with the loss of immnohistochemical labeling. For DPC4/SMAD4, all exons sequenced as wild type, and no abnormalities were detected by MLPA (data not shown). These findings are consistent with retention of expression shown by IHC labeling. The results of cell line IPMN-1T and IPMN-1Asp both matched a non-neoplastic sample from the patient using DNA fingerprinting (Supplementary Table 1).

\section{Tumorigenicity of the IPMN-1 Cell Lines}

In anchorage-independent cloning assays, both IPMN-1Asp and IPMN-1T grew in soft agar and formed colonies at approximately equivalent rates. The frequency of colony formation was significantly less than that of the positive control Panc1 cells (Supplementary Figure S3), but higher than the negative control HPDE cells. We used invasion through matrigel to select an invasive subclone from the population of cells by plating them on top of the matrigel-coated filters, and growing the cultures until cells could be recovered from the bottom of the wells. Using this approach, invasive IPMN$1 \mathrm{~T}$ cells were not detected until after 2 weeks of growth on the matrigel, whereas Pancl invaded by $6 \mathrm{~h}$. Invasive cells were expanded and the matrigel selection process was repeated.

We then tested the parental IPMN-1T, the second passage matrigel-selected derivative (IPMN-1T-M2), and Panc1 in the standard matrigel assay, which measures cell number on the bottom of a matrigel-coated filter at 6-72 h (Supplementary Figure S4). At 6 and $24 \mathrm{~h}$ time points, no cells had invaded from either the IPMN-1T or IPMN-1T-M2 cells, in contrast to the Panc1 cells. After $48 \mathrm{~h}$, a few cells had invaded from both IPMN-1T and IPMN-1T-2M cultures.

In addition, to assess in vivo tumorigenicity, two NOG mice were subcutaneously injected with 20 million cells bilaterally, and one tumor was detected approximately 12 weeks after injection that measured $7 \times 6 \mathrm{~mm}\left(126 \mathrm{~mm}^{3}\right.$, Figure 4a). The tumor formed cysts and papillary structures as typically seen in IPMNs. Histology showed only non-invasive IPMN with high-grade dysplasia, with a pancreatobiliary subtype (Figure $4 \mathrm{~b}$ and $\mathrm{c}$ ). Half of this tumor was subcutaneously re-implanted into a third passage NOG mouse. After 14 weeks, the tumor had grown to $20 \times 15 \mathrm{~mm}$ $\left(2250 \mathrm{~mm}^{3}\right.$, Figure $\left.4 \mathrm{~d}\right)$ and was explanted. The tumor formed a cystic mass, from which we aspirated approximately $4.5 \mathrm{ml}$ of mucinous fluid. After aspirating the cyst fluid, the tumor was opened, and papillary nodules were revealed inside the cystic mass (Figure 4e). The histology (Figure $4 \mathrm{f}$ and g) showed an IPMN-like papillary structure with high-grade dysplasia, but without invasion.

\section{DISCUSSION}

We report that IPMNs can be consistently grown in NOG mice at high frequency $(8 / 10,80 \%)$, including those without an associated invasive component $(5 / 8,62 \%)$. We also successfully established the cell lines, IPMN-1T and IPMN-1Asp, from case 1, possibly because it contained a small invasive component. Tumorigenicity was confirmed by growth in soft agar and tumor production in second and third passage mice. In addition, the $p 16 / C D K N 2 A$ gene was homozygously deleted. The epithelial differentiation of the line was confirmed by the expression of cytokeratin. DNA fingerprinting confirmed the patient from whom it was derived. Matrigel selection of a derivative line, albeit after 2 weeks of growth on the matrigel, suggests that there is a minor subpopulation of cells capable of invasion. This is consistent with the surgical pathology description of the primary tumor. However, we favor the notion that most cells in this mixed culture are noninvasive for the following reasons: because the original tumor histology was a $2.5 \mathrm{~cm}$ IPMN with only microscopic invasion of $<1 \mathrm{~mm}$, as passage of this cell line in NOG mice showed 
tumorigenicity without any invasion, and because the original cell line lacked invasion in the matrigel assay as typically performed.

We attempted to propagate six additional cases in vitro, but were unable to generate cell lines. The failure to generate cell lines fell into three general patterns: first, the neoplastic cells failed to attach; second, the primary cultures were initially successful, but the cell replication was extremely slow, and the cells gradually died; finally, the fibroblasts overgrew the neoplastic cells and destroyed them. To overcome these problems, we prepared a variety of conditions to find the optimal conditions for each culture. Despite the variety of approaches used, the substrate and growth medium may still lack essential growth factors. Finally, it may be impossible to produce cell lines from some IPMNs, because stromal cells may produce essential paracrine growth factors. ${ }^{27}$

We further evaluated two of the available cases that grew in mice using immunohistochemical labeling and DNA sequencing. The patterns of protein expression in the primary tumor and xenograft generally matched. The expression of cytokeratin and vimentin in the cell lines IPMN-1T and IPMN-1Asp matched the expression observed in the paired primary tumor and xenograft samples. Expression of MUC was also consistent between the primary, xenograft, and cell lines. The p16/CDKN2A, TP53, and DPC4/SMAD4 expression patterns matched with their genetic characterizations.

Our work with IPMNs is actively ongoing. First, we are attempting to document the frequency of engraftment and rate of growth of IPMNs in nude, SCID, and NOG mice. Second, we are attempting to isolate invasive and non-invasive components from IPMN-1. In addition, we are continuing our efforts to establish IPMN cell lines from purely non-invasive IPMNs, and plan to use them to screen for chemoprevention agents.

Supplementary Information accompanies the paper on the Laboratory Investigation website (http://www.laboratoryinvestigation.org)

\section{ACKNOWLEDGEMENTS}

We acknowledge Dr Ming Tsao for generously providing the HPDE cell line. We also acknowledge Drs Elizabeth M Jaffee, Robert Anders, and Rajni Sharma for helpful discussions, in addition to Ms Guanglan Mo and Dante Trusty for helpful discussions and technical support. This work was funded, in part, by RO1CA130938 (JRE) and P50CA62924 (PI: Dr Scott Kern), the Stewart Trust, the Michael Rolfe Pancreatic Cancer Foundation, and the Mary Lou Wootton Pancreatic Cancer Research Fund.

\section{DISCLOSURE/CONFLICT OF INTEREST}

The authors declare no conflict of interest.

1. Society AC. Cancer Facts and Figures 2008. American Cancer Society: Atlanta, 2008.

2. Parkin DM, Bray F, Ferlay J, et al. Global cancer statistics, 2002. CA Cancer J Clin 2005;55:74-108.

3. Jemal A, Murray T, Samuels A, et al. Cancer statistics, 2003. CA Cancer Clin 2003;53:5-26.

4. Hruban RH, Pitman MB, Klimstra DS. Tumors of the pancreas. Armed Forces Institute of Pathology, 4th edn. The American Registry of Pathology: Washington, DC, 2007, pp 75-164.
5. Longnecker DS, Adler G, Hruban RH, et al. Introductal PapillaryMucinous Neoplasms of the Pancreas. WHO classification of tumor digestive system, IARC Press: Lyon, France, 2000, pp 237-240.

6. Kimura W, Sasahira N, Yoshikawa T, et al. Duct-ectatic type of mucin producing tumor of the pancreas-new concept of pancreatic neoplasia. Hepatogastroenterology 1996;43:692-709.

7. Nakagohri $\mathrm{T}$, Kenmochi $\mathrm{T}$, Kainuma $\mathrm{O}$, et al. Intraductal papillary mucinous tumors of the pancreas. Am J Surg 1999;178:344-347.

8. Terris $\mathrm{B}$, Ponsot $\mathrm{P}$, Paye $\mathrm{F}$, et al. Intraductal papillary mucinous tumors of the pancreas confined to secondary ducts show less aggressive pathologic features as compared with those involving the main pancreatic duct. Am J Surg Pathol 2000;24:1372-1377.

9. Yamaguchi $K$, Sugitani $A$, Chijiiwa $K$, et al. Intraductal papillarymucinous tumor of the pancreas: assessing the grade of malignancy from natural history. Am Surg 2001;67:400-406.

10. Maitra A, Kern SE, Hruban RH. Molecular pathogenesis of pancreatic cancer. Best Pract Res Clin Gastroenterol 2006;20:211-226.

11. Jones $S$, Zhang $X$, Parsons DW, et al. Core signaling pathways in human pancreatic cancers revealed by global genomic analyses. Science 2008;321:1801-1806.

12. Z'Graggen K, Rivera JA, Compton CC, et al. Prevalence of activating $\mathrm{K}$-ras mutations in the evolutionary stages of neoplasia in intraductal papillary mucinous tumors of the pancreas. Ann Surg 1997;226: 491-498; discussion 498-500.

13. Islam HK, Fujioka Y, Tomidokoro T, et al. Immunohistochemical study of genetic alterations in intraductal and invasive ductal tumors of the pancreas. Hepatogastroenterology 2001;48:879-883.

14. Sasaki $S$, Yamamoto $H$, Kaneto $H$, et al. Differential roles of alterations of p53, p16, and SMAD4 expression in the progression of intraductal papillary-mucinous tumors of the pancreas. Oncol Rep 2003;10:21-25.

15. Biankin AV, Biankin SA, Kench JG, et al. Aberrant p16(INK4A) and DPC4/ Smad4 expression in intraductal papillary mucinous tumours of the pancreas is associated with invasive ductal adenocarcinoma. Gut 2002;50:861-868.

16. Wada K. p16 and p53 gene alterations and accumulations in the malignant evolution of intraductal papillary-mucinous tumors of the pancreas. J Hepatobiliary Pancreat Surg 2002;9:76-85.

17. lacobuzio-Donahue CA, Klimstra DS, Adsay NV, et al. Dpc-4 protein is expressed in virtually all human intraductal papillary mucinous neoplasms of the pancreas: comparison with conventional ductal adenocarcinomas. Am J Pathol 2000;157:755-761.

18. Sahin F, Maitra A, Argani P, et al. Loss of Stk11/Lkb1 expression in pancreatic and biliary neoplasms. Mod Pathol 2003;16:686-691.

19. Schonleben F, Qiu W, Ciau NT, et al. PIK3CA mutations in intraductal papillary mucinous neoplasm/carcinoma of the pancreas. Clin Cancer Res 2006;12:3851-3855.

20. Chari ST, Yadav D, Smyrk TC, et al. Study of recurrence after surgical resection of intraductal papillary mucinous neoplasm of the pancreas. Gastroenterology 2002;123:1500-1507.

21. Salvia R, Fernandez-del Castillo C, Bassi C, et al. Main-duct intraductal papillary mucinous neoplasms of the pancreas: clinical predictors of malignancy and long-term survival following resection. Ann Surg 2004:239:678-685; discussion 685-687.

22. Tanaka M, Chari S, Adsay V, et al. International consensus guidelines for management of intraductal papillary mucinous neoplasms and mucinous cystic neoplasms of the pancreas. Pancreatology 2006;6:17-32.

23. Jaffee EM, Schutte M, Gossett J, et al. Development and characterization of a cytokine-secreting pancreatic adenocarcinoma vaccine from primary tumors for use in clinical trials. Cancer J Sci Am 1998;4:194-203.

24. Furukawa T, Duguid WP, Rosenberg $L$, et al. Long-term culture and immortalization of epithelial cells from normal adult human pancreatic ducts transfected by the E6E7 gene of human papilloma virus 16 . Am J Pathol 1996;148:1763-1770.

25. Lieber M, Mazzetta J, Nelson-Rees W, et al. Establishment of a continuous tumor-cell line (panc-1) from a human carcinoma of the exocrine pancreas. Inter J Canc 1975;15:741-747.

26. Furukawa T, Kloppel G, Volkan Adsay N, et al. Classification of types of intraductal papillary-mucinous neoplasm of the pancreas: a consensus study. Virchows Arch 2005;447:794-799.

27. Ricci F, Kern SE, Hruban RH, et al. Stromal responses to carcinomas of the pancreas: juxtatumoral gene expression conforms to the infiltrating pattern and not the biologic subtype. Cancer Biol Ther 2005;4:302-307. 